\title{
Diagnostic medical ultrasound awareness among women in Sub-Saharan Africa (SSA)
}

\begin{abstract}
US is vital for the health care of women. SSA countries still have low level of awareness of this tool among women. There is limited information in SSA and most studies focus on prenatal US. Many factors such as level of education, cost of purchasing and training in US, low level of development, government policies, training of medical health care workers, insufficient infrastructures are all seen to influence the knowledge of SSA women on US. This knowledge gap motivated the writing of this editorial whose main aim was to assess US awareness among SSA women, with hopes that its outcome would positively influence policies in SSA countries on US use among Women.
\end{abstract}

Keywords: SSA, women, infrastructures, x- rays, gamma rays, alpha particles, neutrons
Volume 4 Issue 6 - 2017

\author{
Samuel Nambile Cumber,' Keneth Nkeh \\ Nchanji ${ }^{2}$ \\ 'Department of Nursing \& Public Health, University of Kwazulu- \\ Natal, South Africa \\ ${ }^{2}$ St Louis University Institute of health and Biomedical Science \\ Bamenda, Cameroon
}

Correspondence: Samuel Nambile Cumber, Discipline of Public Health Medicine, Department of Nursing \& Public Health, College of Health Sciences, University of Kwazulu-Natal, Durban, South Africa, Email samuelcumber@yahoo.com

Received: September 20, 2017 | Published: December 21,

2017

\section{Introduction}

Medical Imaging is indispensable for Medical Practice in today's world. It embodies various Imaging modalities, with Ultrasonography (also known as Ultrasound) being one of them. Ultrasonography is a medical diagnostic Imaging modality, which uses sound waves of frequency greater than 20 Kilohertz $(20 \mathrm{KHz})$. Sound waves are non-ionizing radiations unlike $\mathrm{X}$ - Rays, gamma rays, alpha particles, or neutrons. Ultrasound is generated with the help of the ultrasound machine, using the piezo-electric principle. ${ }^{1}$

Ultrasound use in women is dominant in the areas of Obstetrics and Gynecology, breast imaging, and also musculoskeletal, thoracic and abdominal imaging. Women can do an US scan to diagnose lumps in the breast, to check and evaluate pregnancy (obstetrics), which is the most widely applicable in women, ${ }^{2-6}$ to evaluate the pelvic organs and the related structures in the pelvis (gynecology). ${ }^{7,8}$ The benefits and rational for the wide advocate of US use in women is due to the following: US use is relatively cheap and easily affordable and accessible, Ultrasound scans are done in real time, there is no use of ionizing radiations such as X-Rays with side effects, sectional images obtained give detailed information about the structures scanned, requires minimal patient preparations such as a filled urinary bladder for pelvic US and will also effectively diagnose soft tissue problems better than two dimensional plain radiography, ${ }^{1}$ besides its effectiveness in detecting an ectopic pregnancy. ${ }^{3}$

Despite these numerous benefits of US in women, studies reveal that Ultrasound awareness among women in Sub-Saharan Africa still remains low; ;,5,6 as most women reported not haven heard of US, besides this, those who come for prenatal US scans usually are mainly interested in knowing the sex of their baby (this shows that majority are not properly schooled). ${ }^{2}$ This ignorance among women cuts across many Sub-Saharan African countries. ${ }^{6}$ This explains the motivation for this editorial whose objective is to assess the ultrasound awareness among these women of SSA. It is anticipated that the findings of this study will stimulate decision makers of SSA to influence Ultrasound Use among women positively.

\section{General situation of diagnostic medical ultra- sound services in sub-saharan Africa}

Ultrasound services are rarely available in most communities of SSA countries. ${ }^{6}$ Even at the level of some health facilities, midwives, who are most connected to women, show some level of ignorance on its value in women. ${ }^{4}$ In some SSA countries however, the proliferation of such services without appropriate regulation is seen to be a problem. ${ }^{2}$ The associated cost of acquiring US equipment, the training of the professionals and availability also contribute to the ignorance of women in SSA. ${ }^{3,4}$ Women in the rural areas are mostly deprived of its services, thus there is need for their inclusion. Reports on a similar issue revealed that, radio announcements and programs are good tools to educate women on US besides ANC visits.

\section{Current level of ignorance on us use and as- sociated factors}

Based on a study conducted by Joshua et al., ${ }^{9}$ out of a total of 200 women who were interviewed, 33 respondents $(16.8 \%)$ on a total of 196 declared that they have never done an US scan, $19(58.4 \%)$ on a total of 190 confessed having received no information on what Ultrasound is all about. 34 women considered Ultrasound as unsafe as they believe US uses X-rays which can cause cancer, this really reveals that most women in SSA are really ignorant of US. It is equally worth noting that in this study, most women were within the age group of 25-29years. ${ }^{9}$ Similar findings in other SSA countries show that most women especially pregnant needed US mainly for sex determination and general fetal assessment, ${ }^{2,10}$ and in most cases, the need for the exam was initiated by health care workers, not the women themselves. ${ }^{2}$ Data is limited on US awareness among women in SSA, the few available are focused on Prenatal US. The gross level of unawareness has potential consequences such as ectopic pregnancies, still births, infertility due to fibroids in women's uterus, breast cancer, broken relationships, frustrations and unnecessary operations in these women and in some cases death. ${ }^{3}$ 
Many factors account for this ignorance. Obstetricians and Gynecologists who request most US exams, do not properly educate these women, most Nurses and Midwives who frequently interact with women are equally ignorant of the value of US, partly due to the nature and design of most of their educational curricula which do not include courses in Obstetrics and Gynecological Ultrasound, ANC services offered in Cameroon and many SSA countries hardly lay emphasis on. ${ }^{3,4}$ Moreover, most government programs concerning women hardly emphasize the value of US, which partly may be due to negligence especially on Medical Imaging Training in most SSA countries, an aspect that could boast US awareness among women and enable training of trainers. ${ }^{3,4}$

\section{Filling the knowledge gap}

To address this issue, medical students and Nursing/Midwifery students should be taught Ultrasonography as a major course, so they could help educate women. ${ }^{4}$ Women during ANC and PNC visits should be given lectures on Ultrasonography by Sonographers, sensitization programs on popular TV programs, ${ }^{5}$ focus on the value of US, educative fly outs and leaflets on US, dedicated people should be sent to Women's groups such as "Njangis", the government through the Ministry of women empowerment of SSA countries could sponsor peer educators on Ultrasonography training, Sonographers from Medical Imaging Training schools should be recruited and deployed to health centers so they can better educate women especially those in the villages and remote areas, screening programs for women could be done, during which US scans can be done, and this will serve as a window to educate the women, such programs should also be made sustainable by the government and NGOs, for every given intervention, regular evaluation of progress should be done to check the level of awareness and through the wide use of the social networks such as MTN, Nextel, Orange, regular messages can be given to the women and the general population on the value of Ultrasound in women, such programs could be co-sponsored by the Ministries of public Health and Women Empowerment and the Family.

\section{Conclusion and recommendations}

Detail research should be conducted to evaluate the general awareness of women on US.

In a nut shell, if the above solutions are implemented most women will be aware of US and benefit from the use of this very useful Medical
Diagnostic Imaging Modality and overall health improvement will be achieved.

\section{Acknowledgements}

None.

\section{Conflict of interest}

Author declares that there is no conflict of interest.

\section{References}

1. Kathryn AG. Ultrasound in Obstetrics and Gynaecology: A Practitioner's Guide. Pasadena, California: Davis Publishing Inc; 2014.

2. Christopher AE, Imran OMB, Anthony OM, et al. Client's Reasons for Prenatal Ultrasonography in Ibada. South West of Nigeria. BMC Women's Health. 2009;9:12.

3. Adrian HF, Sherim K, Gloria YW, et al. Frequency of Utilization of U1trasound in the Diagnosis of Ectopic Pregnancies in Sub Saharan African Countries: A Systemic Review. African Journal of Emergency Medicine. 2015;5(1):31-36.

4. Christina K, Michael K, Tonny T, et al. Addressing Obstetrical Challenges at 12 Rural Ugandan Health Facilities: Findings from an International Ultrasound and Skills Development Training for Midwives in Uganda. Int J MCH AIDS. 2016;5(1):46-52.

5. William C, Geoffrey A, Christopher M, et al. Effectiveness of Advertising Availability of Prenatal Ultrasound on Uptake of Antenatal Care in Rural Uganda: A Cluster Randomized Trial. PLOS ONE. 2017.

6. Dorothy AO, Nancy M, Bryn K, et al. 'You Cannot Know if it's a Baby or not a Baby': Uptake, Provisions and Perceptions of Antenatal Care and Routine Antenatal Ultrasound Scanning in Rural Kenya. BMC Pregnancy and Child Birth. 2015;15:127.

7.www.cancer.org

8. Hofmeyr GJ. Routine Ultrasound in Early Pregnancy: Is it Worthwhile in Low Income Countries? Ultrasound Obstet Gynecol. 2009;34(4):367-370.

9. Joshua T, Boniface M, Odile FZ, et al. Routine Prenatal Ultrasonography: The Pregnant Women's Expectations and Safety in a Central African Obstetric Population. Open Journal of Obstetrics and Gynaecology. 2013;3(7):573-576.

10. Pauline Rossi, Lea Rouanet. Gender Preferences in Africa: A Comparative Analysis of Fertility Choices. World Development. 2014;72:326-345. 